\title{
X-rays, $\gamma$-rays and neutrinos from collisionless shocks in supernova wind breakouts
}

\author{
Boaz Katz ${ }^{1}$, Nir Sapir ${ }^{2}$ and Eli Waxman ${ }^{2}$ \\ ${ }^{1}$ Institute for Advanced Study, Princeton, NJ 08540, USA \\ email: boazka@ias.edu \\ ${ }^{2}$ Dept. of Particle Phys. \& Astrophys., Weizmann Institute of Science, Rehovot 76100, Israel \\ email: nir.sapir@weizmann.ac.il \\ email: eli.waxman@weizmann.ac.il
}

\begin{abstract}
Some of the observed bursts of X-rays/Gamma-rays associated with supernovae $(\mathrm{SNe})$ as well as very luminous SNe may result from the breakout of the SN shock from an optically thick wind surrounding the progenitor. We show that in such scenarios a collisionless shock necessarily forms during the shock breakout. An intense non-thermal flash of $\lesssim 1 \mathrm{MeV}$ gamma rays, hard $\mathrm{X}$-rays and multi-TeV neutrinos is produced simultaneously with and following the typical soft X-ray breakout emission, carrying similar or larger energy than the soft emission. The non-thermal flash is detectable by current X-ray telescopes and may be detectable out to 10's of Mpc by km-scale neutrino telescopes.
\end{abstract}

Keywords. (stars:) supernovae: general, shock waves, radiation mechanisms: nonthermal

\section{Introduction}

One of the most exciting, relatively recent, developments in the study of SNe, is the association with type Ib/c SNe of bursts of hard X-rays/ $\gamma$-rays, lasting for tens to thousands of seconds (e.g. Galama et al. 1998, Hjorth et al. 2003, Malesani et al. 2004, Campana et al. 2006, Soderberg et al. 2008, Vergani et al. 2010).

What are these bursts? A list of the confirmed associations, including some of the main observable properties of the bursts, is given in table 1. Perhaps the most important feature of these bursts is that they are different from one another by several orders of magnitude in energy, time scale and rate. In addition, most of these bursts must be very rare compared to SNe. To allow a rough estimation of the typical rates, the volume in which each of these bursts was detected is provided in the 4 th column of the table. With the exception of SN1998bw and SN2008D, 100-10000 type Ibc SNe have occurred in each of these volumes within the typical $10 \mathrm{yr}$ lifetime of the relevant $\gamma$-ray detectors (assuming a conservative low rate of SNe Ibc of $10^{-5} \mathrm{Mpc}^{-3} \mathrm{yr}^{-1}$ ) with only $\sim 1$ X-ray/gamma-ray event. The extremely bright radio afterglow of SN1998bw, implies that this too is a rare event (e.g. Soderberg et al. 2010). The only exception left is the burst associated with 2008D, which could be common among Ib SNe (e.g. Mazzali et al. 2008).

The huge differences in energy, time scale and rate of these events implies that the physical processes involved in different bursts may be very different. In particular, the exciting fact that the burst properties of SN2003dh are similar to cosmological gammaray bursts (GRBs) does not imply that the other (much weaker and common) events are related to cosmological GRBs.

It has long been suggested that an intense burst of soft X-ray radiation is expected to be emitted at the initial phases of every SN explosion, once the radiation mediated shock 
Table 1. 'GRB/XRF'-SNe

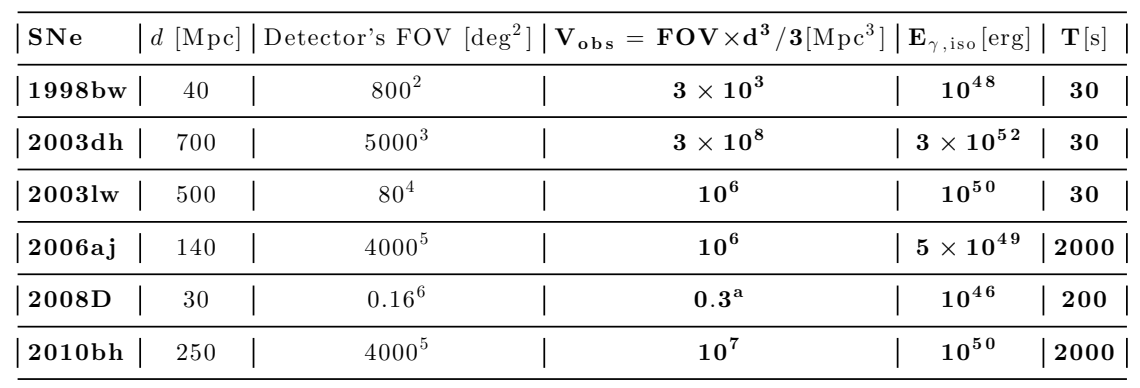

Notes:

1 Data taken from Fan et al. (2011), except for SNe 2008D which is taken from Soderberg et al. (2008) Burst detected by ${ }^{2}$ BeppoSax, ${ }^{3}$ HETE-II, ${ }^{4}$ Integral, ${ }^{5}$ Swift-BAT, ${ }^{6}$ Swift-XRT

a This small volume does not represent the rate since there are $\ll 1$ galaxies on average within this volume, while the detector is focused on galaxies most of the time

reaches the edge of the star (e.g. Colgate 1974, Falk et al. 1978). It is natural to study the possibility that some of these events are related to such breakouts and several such claims have been made based on the bursts properties (e.g. Kulkarni et al. 1998, Campana et al. 2006, Soderberg et al. 2008). If detected, the properties of the shock breakout burst allow a robust determination of important progenitor properties including its radius.

The main challenge to the association of these events with the predicted properties of shock breakouts is that their spectrum is much harder than expected. The spectrum of typical shock breakouts is expected to be soft, sharply declining beyond a few hundreds of eV's (e.g. Falk et al. 1978, Ensman \& Burrows 1991, Matzner \& Mckee 1999), in contrast to the observed hard spectrum extending to multi-keV energies in all of the observed bursts.

This concern is relaxed by the results of radio and X-ray afterglow observations on scales of days-months which showed the presence of fast material $\Gamma \beta \gtrsim 0.2-1$, carrying energies similar to the burst energies. At these inferred fast shock velocities, departure from equilibrium may imply very high electron temperatures, reaching tens or hundreds of $\mathrm{keV}$ (Weaver 1976, Katz et al. 2010) which naturally account for the hard spectra. Note that the large amount of energy carried by very fast $(\Gamma \beta \sim 1)$ ejecta (with the exception of SN2008D) poses a challenge in itself to SNe explosion models. SN2008D, which does not suffer the latter problem and could be a relatively common phenomenon among SNe, is a particularly appealing shock breakout candidate.

A second challenge is that the progenitor radius inferred from the breakout interpretation, $R \approx 10^{12}\left(E_{\gamma} / 10^{47} \mathrm{erg}\right)^{1 / 2} \beta^{-1 / 2} \mathrm{~cm}$ (e.g. Matzner \& McKee 1999, Katz et al. 2012), is larger than the expected radius $R \lesssim 10^{11} \mathrm{~cm}$ of WR progenitors believed to produce Ibc SNe, which agrees with inferred radii from followup UV and optical observations (e.g. Campana et al. 2006, Soderberg et al. 2008, Rabinak \& Waxman 2011).

If the star is surrounded by a sufficiently optically thick shell of circumstellar matter (CSM), e.g. a high density wind, the breakout occurs within the shell at radii much larger than the progenitor and may solve this problem. Several observed $\gamma$-ray/X-ray flashes associated with SNe have been suggested to be such cases (Tan et al. 2001, Campana et al. 2006, Soderberg et al. 2008).

Breakout outbursts of slower shocks, $v_{\mathrm{sh}} \sim 0.03 c$, have been suggested to account for strong optical/UV transients (Ofek et al.2010) and very luminous SNe (e.g. Quimby et al. 2007, Smith et al. 2007, Miller et al. 2009). In order to explain the high energy (reaching $10^{51} \mathrm{erg}$ ) emitted in these SNe, CSM parameters were suggested such that the 
diffusion time scale through the CSM is comparable to the dynamical time scale, $R / v_{\text {sh }}$ (e.g. Quimby et al. 2007, Smith et al. 2007b, Moriya et al. 2011, Chevalier \& Irwin 2011, contribution to this proceedings by T. Moriya). If true, the observed emission is, by construction, the breakout outburst from the CSM.

Following breakout, the radiation mediated shock is expected to become a collisionless shock, leading to the emission of gamma-rays and neutrinos (e.g. Waxman \& Loeb 2001). In the absence of a (significant) wind, the small mass of the shell shocked by the collisionless shock implies that only a small fraction, $\lesssim 10^{-2}$, of the breakout energy is converted to such high energy radiation. Moreover, the formation of a collisionless (or collisional) shock is controversial (e.g. Klein \& Chevalier 1978, Epstein 1981, Sapir et al. 2011), since the light shell may be accelerated to sufficiently high velocity by the escaping radiation.

In this letter we show that if the progenitor is surrounded by an optically thick CSM, e.g. a dense wind, a collisionless shock is necessarily created during the breakout, and that an energy comparable to or greater than the breakout energy is emitted by quasi-thermal particles in high energy ( $\gtrsim 50 \mathrm{keV})$ photons, and by accelerated protons in high energy $(\gtrsim 1 \mathrm{TeV})$ neutrinos. The latter is an extension of the study of high energy emission from the interaction of the ejecta with a dense optically thin CSM (Murase et al. 2010).

\section{Formation of a collisionless shock}

Consider for simplicity a piston moving with a constant velocity $v=10^{9} \mathrm{~cm} \mathrm{sec}^{-1} v_{9}$ through an optically thick fully ionized hydrogen wind with a density profile

$$
\rho(r)=\frac{c}{v} \frac{m_{p}}{\sigma_{T} R_{\mathrm{br}}}\left(r / R_{\mathrm{br}}\right)^{-2},
$$

where $R_{\mathrm{br}}=10^{14} R_{14} \mathrm{~cm}$ is a normalization parameter with dimensions of length. A shock propagates ahead of the piston with velocity $v_{\mathrm{sh}} \sim v$. As long as the optical depth across the shock transition region, $\Delta_{\tau, \mathrm{sh}} \sim c / v$, is much smaller than the optical depth of the system, $\Delta_{\tau}=(c / v) R_{\mathrm{br}} / r$, the post-shock radiation is confined. Once the shock reaches $r \sim R_{\mathrm{br}}$, the width of the shock becomes comparable to the size of the system and a significant fraction of the post-shock energy can be emitted during one dynamical time scale, $R_{\mathrm{br}} / v$. This emission is the breakout outburst discussed above.

The material lying ahead of the piston must be accelerated to velocities approaching $v$ by some process. At large optical depth, where the radiation mediated shock is sustained, the radiation accelerates the material by Compton scattering off the electrons. The maximal velocity to which a fluid shell can be accelerated by this process is given by

$$
v_{\max }=\frac{E_{\gamma} / c}{4 \pi r^{2}} \frac{\sigma_{T}}{m_{p}},
$$

where $E_{\gamma}=\int L_{\gamma} d t$ is the radiation energy emitted through the fluid shell and $r$ is its initial position. This maximum velocity is achieved if all of the flowing photons move radially and the shell does not expand considerably during the passage of the radiation. In this case, a fraction $\sigma_{T} /\left(4 \pi r^{2}\right)$ of the momentum $E_{\gamma} / c$ carried by the radiation is transferred on average to each proton.

$E_{\gamma}$ is limited by the thermal energy accumulated in the post shock region which, in turn, is limited by $0.5 M(r) v^{2}$, where $M(r)=4 \pi(c / \kappa v) R_{\mathrm{br}} r$ is the wind mass inward of $r$. Using equation (2.2) an upper limit for $v_{\max }, v_{\max }<0.5\left(R_{\mathrm{br}} / r\right) v$, is obtained. This implies that beyond a radius of $0.5 R_{\mathrm{br}}$ the shock can no longer be mediated by radiation and must be transformed into a collisional or a collisionless shock. Since the 
ion plasma frequency, $\omega_{p}=\left(4 \pi \rho e^{2} / m_{p}^{2}\right)^{1 / 2} \sim 10^{9} v_{9}^{-1 / 2} R_{14}^{-1 / 2} \sec ^{-1}$, is many orders of magnitudes larger than the ion Coulomb collision rate per particle, $\nu_{C}=\rho \sigma_{C} v / m_{p} \sim$ $2 \times 10^{-2} R_{14}^{-1} v_{9}^{-4} \sec ^{-1}$ (e.g. Waxman \& Loeb 2001), the shock will be collisionless, i.e. mediated by collective plasma instabilities.

\section{Emission from thermal electrons.}

The collisionless shock heats the protons on a time scale of $\omega_{p}^{-1}$ to a temperature roughly given by

$$
T_{p} \sim \frac{3}{16} m_{p} v^{2} \sim 0.4 v_{9}^{2} m_{e} c^{2} \sim 0.2 v_{9}^{2} \mathrm{MeV}
$$

The electron temperature depends on the unknown amount of collisionless heating. A lower limit for the electron temperature can be obtained by assuming that there is no collisionless heating.

The collisional heating rate of the electrons due to Coulomb collisions with the protons is given by

$$
-\frac{d T_{p}}{d t}=\frac{d T_{e}}{d t} \approx \lambda_{e p} \sqrt{\frac{2}{\pi}} \frac{m_{e}}{m_{p}} T_{p}\left(\frac{T_{e}}{m_{e} c^{2}}\right)^{-3 / 2} n_{d} \sigma_{T} c,
$$

where $\lambda_{e p}=30 \lambda_{e p, 1.5}$ is the Coulomb logarithm and it was assumed that $T_{e} /\left(m_{e} c^{2}\right) \gg$ $T_{p} /\left(m_{p} c^{2}\right)$. The fastest possible cooling source for thermal electrons is Inverse Compton (IC) scattering of the local radiation field, which carries a significant fraction $\epsilon_{\gamma} \lesssim 1$ of the post shock energy and is given by

$$
\frac{d T_{e}}{d t}=-\frac{2}{3} \frac{4 T_{e}}{m_{e} c^{2}} \sigma_{T} c U_{\gamma}
$$

where $U_{\gamma}=\epsilon_{\gamma} n_{d} T_{d}$ is the photon energy density and $n_{d}$ is the shocked material proton density. Assuming $U_{\gamma} \lesssim n_{d} T_{p}$ (equivalently, $\epsilon_{\gamma} \lesssim 1$ ) we find

$$
\frac{T_{e}}{m_{e} c^{2}} \gtrsim 0.6\left(\frac{m_{e}}{m_{p}} \lambda_{e} / \epsilon_{\gamma}\right)^{2 / 5} \Rightarrow T_{e} \gtrsim 60 \mathrm{keV} \lambda_{e p, 1.5}^{2 / 5} .
$$

The time it takes the protons to lose a significant fraction of their energy (which is of the order of the total available energy) is

$$
\begin{aligned}
t_{\mathrm{p}} & =T_{p}\left(\frac{d T_{p}}{d t}\right)^{-1} \lesssim 0.6\left(\lambda_{e p} \frac{m_{e}}{m_{p}}\right)^{-2 / 5} \epsilon_{\gamma}^{-3 / 5}\left(n_{d} \sigma_{T} c\right)^{-1} \\
& \sim 3 \lambda_{e p, 1.5}^{-2.5} \epsilon_{\gamma}^{-3 / 5}\left(n_{d} \sigma_{T} c\right)^{-1} .
\end{aligned}
$$

The proton cooling time is thus much shorter than the dynamical time $R_{\mathrm{br}} / v=(c / v)^{2} /$ $\left(n \sigma_{T} c\right)$, where $n=\rho / m_{p}$ is the proton number density in the pre-shocked region and is smaller than $n_{d}$ by the compression factor. This is not surprising. While the shock is radiation mediated, radiation energy equal to the mechanical energy is generated on each shock crossing time scale. At breakout, the shock crossing time scale equals the dynamical scale and radiation with energy density comparable to the total energy density must be generated during the dynamical time scale. In fact, since the electron temperature is higher than that expected in a corresponding radiation mediated shock, the emission efficiency is even higher.

The shock is strongly radiative and the energy is efficiently converted to radiation. The typical photon energies are expected to be of the same order of magnitude as the 
electron energies, i.e. $\gtrsim 60 \mathrm{keV}$. The calculation of the emitted spectrum is beyond the scope of this paper. We note that since the initial photon energies are much lower $(\sim 1 \mathrm{eV}$ assuming equilibrium) we expect that the spectrum hardens continuously with time and that on the breakout time scale, significant emission is likely emitted at all intermediate energies.

We conclude that gamma-rays/hard X-rays will be emitted with total energy comparable to that of the breakout energy

$$
E_{\gamma}=\frac{4 \pi R_{\mathrm{br}}^{2}}{\sigma_{T}} m_{p} c v \sim 10^{49} v_{9} R_{14}^{2} \mathrm{erg}
$$

on a time scale similar to the breakout time $t \sim R / v \sim 1 R_{14} v_{9}^{-1} \mathrm{~d}$ with typical luminosity

$$
L_{\gamma} \sim 10^{45} R_{14} v_{9}^{2} \operatorname{erg~sec}^{-1} .
$$

When the shock expands, it will remain radiative beyond $R_{\mathrm{br}}$ and the total emitted energy, integrated over longer time scales, may be significantly larger than that of the breakout energy.

\section{Accelerated protons: Non-thermal emission energy.}

Relativistic particles (CRs) accelerated in the collisionless shock that forms due to the collision of the SN ejecta with dense interstellar material may emit high energy gamma rays and neutrinos due to the interaction with the dense material (Murase et al. 2010). The collisionless shock that was shown above to be produced during breakout from a dense wind is a constrained example of such interaction and may be a source of detectable high energy neutrinos and gamma rays. Here we focus on the emission from accelerated protons and their products. In what follows it is assumed that the accelerated protons carry a fraction $\epsilon_{C R}=0.1 \epsilon_{C R,-1}$ of the post shock energy and have a flat power law energy distribution, $\varepsilon^{2} d n / d_{\varepsilon} \propto \varepsilon^{0}$.

The cooling time of a relativistic accelerated proton due to inelastic pp collisions is roughly given by

$$
\begin{aligned}
t_{p p} & =\left(0.2\left(\rho / m_{p}\right) \sigma_{p p} c\right)^{-1}=5 \frac{\sigma_{T}}{\sigma_{p p}}\left(\frac{v}{c}\right)^{2} \frac{R_{\mathrm{br}}}{v} \\
& \sim 0.1 v_{9}^{2} \frac{R_{\mathrm{br}}}{v} .
\end{aligned}
$$

Hence, for slow enough shock velocities, $v / c \lesssim 0.1$, protons accelerated at breakout efficiently convert their energy to neutrinos, gamma-rays and pairs by pion production and decay (and muon decay). In this section we restrict the discussion to $v / c \lesssim 0.1$. For such shock velocities, the amount of energy emitted by relativistic protons during breakout is expected to be roughly a fraction $\epsilon_{C R}$ of the energy emitted by the thermal particles. Using Eq. (3.6) we have $E_{\mathrm{Non}-\text { thermal }} \sim 10^{48} \epsilon_{C R,-1} R_{14}^{2} v_{9}$ erg.

At later stages, $t_{p p} v / r$ grows linearly with $r$, and as long as it is smaller than unity, the energy converted into pions increases linearly with the accumulated mass. The radius $r_{p p}$ at which the proton energy loss time is equal to the dynamical time, $t_{p p} v / r_{p p}=1$, is

$$
r_{p p} \sim 10 v_{9}^{-2} R_{\mathrm{br}}
$$

Beyond this radius, the fraction of energy converted to pions drops like $1 / r\left(t_{p p} \propto \rho^{-1} \propto\right.$ $r^{2}$ while the available energy increases linearly with $r$ ) implying a logarithmic increase in the total emitted energy. Given that in reality, $v(r)$ is slowly declining, the total 
contribution to the non thermal fluence from $r>r_{p p}$ is of order unity compared to fluence produce up to this radius. The total emitted energy is therefore given by

$$
E_{\text {Non-thermal }} \sim 10^{49} \epsilon_{\mathrm{CR},-1} R_{14}^{2} v_{9}^{-1} \text { erg. }
$$

\subsection{Accelerated protons: Maximal proton energy.}

The maximal proton energy is limited by the time available for acceleration which is the shorter of the dynamical time and energy loss time. The acceleration time depends on the unknown magnetic field value and the loss time depends on the unknown target photon energy distribution. Nevertheless, we next demonstrate that protons are very likely to be accelerated to at least multi-TeV energies.

Assuming Bohm diffusion, the acceleration time to energy $\varepsilon$ is given by

$$
t_{\mathrm{acc}}=\frac{\varepsilon}{(v / c)^{2} e B c} \sim 2 \times 10^{-7} \frac{\varepsilon_{\mathrm{TeV}}}{\epsilon_{B}^{1 / 2} v_{9}^{3 / 2} R_{14}^{1 / 2}} \frac{R_{\mathrm{br}}}{v},
$$

where $B$ is the post shock magnetic field and $\epsilon_{B}=B^{2} /\left(8 \pi \rho v^{2}\right)$ is roughly the fraction of postshock energy carried by it. For $\mathrm{TeV}$ CRs, the acceleration time is thus much shorter than the dynamical time and the $p p$ energy loss time. For protons in the range $10-1000 \mathrm{TeV}$ the strongest possible cooling mechanism is photo-production of pions, with cooling time

$$
t_{p \gamma}=\left(0.2 n_{\gamma} \sigma_{p \gamma} c\right)^{-1} \gtrsim 5 \frac{\sigma_{T}}{\sigma_{p \gamma}} \frac{h \nu_{\gamma}}{m_{p} c^{2}} \frac{R_{\mathrm{br}}}{v},
$$

where $n_{\gamma}\left(h \nu_{\gamma}\right)$ is the target photon number density (typical energy) and we conservatively assumed that $n_{\gamma}=\rho v^{2} /\left(h \nu_{\gamma}\right)$. Photo-production of pions occurs if the proton energy is higher than the threshold, $\sim m_{\pi} m_{p} c^{4} /\left(h \nu_{\gamma}\right) \sim 0.13\left(h \nu_{\gamma} / \mathrm{MeV}\right)^{-1} \mathrm{TeV}$. The possible presence of many $\sim 1 \mathrm{MeV}$ photons implies that photo-production may be important for $1 \mathrm{TeV}$ protons. Photo production is not important if the target photons have $\sim 1 \mathrm{eV}$ energies, as assumed in (Murase et al. 2011). Given the constraint $n_{\gamma} \lesssim \rho v^{2} /\left(h \nu_{\gamma}\right)$, the strongest losses for protons of energy $\varepsilon=\varepsilon_{\mathrm{TeV}} \mathrm{TeV}$ occurs for target photons having a typical energy of $h \nu_{\gamma} \sim m_{\pi} m_{p} c^{4} / \varepsilon \sim 0.13 \mathrm{MeV} \varepsilon_{\mathrm{TeV}}^{-1}$. Using this in Eq. (4.5) we obtain

$$
t_{p \gamma} \gtrsim 1 \varepsilon_{\mathrm{TeV}}^{-1} \frac{R_{\mathrm{br}}}{v} .
$$

Comparing Eq. (4.6) to Eq. (4.4) we conclude that acceleration to multi TeV energies is possible for $\epsilon_{B} \gtrsim 10^{-13} v_{9}^{-3} R_{14}^{-1} \varepsilon_{\mathrm{TeV}}^{4}$, implying that reaching energies well above $1 \mathrm{TeV}$ is very likely.

We verified that proton CRs do not suffer significant losses due to Inverse Compton and Synchrotron emission during the acceleration time, and that the resulting pions and muons do not suffer significant energy losses due to these processes before decaying. Finally, note that the maximal proton energy is increasing with radius since the ratio of proton acceleration time to dynamical time is independent of radius $\left(t_{\text {acc }} \propto B^{-1} \propto\right.$ $\left.\rho^{-1 / 2} \propto r\right)$ while the ratio of all the loss times to the dynamical time decreases with radius.

\subsection{Accelerated protons: Multi TeV neutrinos.}

Roughly a third of the non thermal energy Eq. (4.3) will be emitted in muon neutrinos (and anti-neutrinos) and a significant fraction of this energy may be emitted beyond $\mathrm{TeV}$ energies. In the neutrino energy range of one to hundred $\mathrm{TeV}$, the effective area for muon neutrinos of a Cherenkov neutrino detector like IceCube is increasing linearly with 
energy, approximately as $10^{-6} \varepsilon_{\mathrm{TeV}} A$, where $A=10^{10} A_{10} \mathrm{~cm}^{2}$ is the geometrical crosssection of the detector. The number of muons induced by one to hundred $\mathrm{TeV}$ neutrinos is therefore independent of the neutrino spectrum in this range and is given by

$$
N_{\mu} \sim 5 \frac{E_{\nu_{\mu}, 1-100 \mathrm{TeV} / 10^{51} \mathrm{erg}}}{(d / 100 \mathrm{Mpc})^{2}} \sim 1 \frac{\epsilon_{p,-1} R_{15}^{2}}{v_{9}(d / 100 \mathrm{Mpc})^{2}}
$$

where $d$ is the distance to the $\mathrm{SN}$ and where we optimistically assumed that $1 / 3$ of the non thermal emission, Eq. (4.3), is in multi TeV neutrinos.

\subsection{Accelerated protons: Gamma-rays.}

High energy gamma-rays and pairs with energies reaching multi TeV energy will be generated with a comparable rate to that of the neutrinos. The pairs will emit further high energy gamma-rays by Inverse Compton interactions with the radiation field. Emission below $\sim 1 \mathrm{MeV}$ will be mixed with the emission from the thermal electrons. Emission at a photon energy $h \nu \gtrsim \mathrm{MeV}$ may be suppressed by the large optical depth for pair creation, which depends on the density of photons with energies above the pair production threshold $h \nu_{T} \gtrsim m_{e} c^{2} /(h \nu)$.

An upper limit to the optical depth for pair creation at a given photon energy, $h \nu$, can be obtained by using the fact that the total energy density of photons of any frequency is smaller than $\rho v^{2}$. Assuming that the energy of photons per unit logarithmic frequency does not exceed $\epsilon_{0.1} \times 10 \%$ of $\rho v^{2}$, and focusing on the radius $10 R_{\mathrm{br}}$ at which the protons are still efficiently cooled (for $v \sim 10^{9} \mathrm{~cm} \mathrm{sec}^{-1}$, see Eq. (4.2)), we find

$$
\tau_{\gamma \gamma} \lesssim \epsilon \frac{v}{c} \frac{m_{p}}{m_{e}} \frac{h \nu}{m_{e} c^{2}} \sim \epsilon_{0.1} 0.6 v_{9} \frac{h \nu}{m_{e} c^{2}} .
$$

The emitted spectrum is suppressed by at most $\sim \tau^{-1}$. In this 'worst case scenario', such bursts will be too faint to be observable by high energy ( $h \nu \sim 1 \mathrm{GeV})$ gamma-ray detectors such as Fermi.

\section{Discussion}

We have shown that shock breakouts in optically thick winds will necessarily be accompanied by high energy radiation from a collisionless shock that inevitably forms on the time scale of the breakout outburst.

Low luminosity GRBs associated with SNe have been suggested to be the outbursts associated with fast shocks $v \gtrsim 0.1 \mathrm{c}$ breaking out of dense optically thick winds. As we have shown here, a significant fraction of the observed radiation, or even most of it, may be generated by the collisionless shock that will form during the breakout.

If the slow, $v / c \sim 0.03$, breakout interpretation of events such as PTF09u (Ofek et al. 2010 ) is correct, a significant amount of energy, $E \sim 10^{51} \mathrm{erg}$, is expected to be emitted in hard $\mathrm{X} / \gamma$-rays reaching energies $h \nu \gtrsim 50 \mathrm{keV}$, Eq. (3.4), and multi-TeV neutrinos (see also Murase et al. 2010). X-rays at lower energies are likely to be emitted with similar efficiency and would be easily detected by instruments like the X-ray telescope (XRT; Burrows et al. 2005) on board Swift (Gehrels et al. 2004) or the Chandra X-ray observatory. We note that more detailed followup analysis suggests that the hard X-ray signal may be partly absorbed (Chevalier \& Irwin 2012) or significantly delayed (Svirski et al. 2012, contribution to this proceedings by Ehud Nakar) due to the interaction of the radiation with the thick wind. $\mathrm{TeV}$ neutrinos may be detectable by experiments like IceCube, see Eq. (4.7), provided such events are sufficiently common and a similar event occurs at a distance $d \lesssim 100 \mathrm{Mpc}$ (compared to $\sim 300 \mathrm{Mpc}$ for PTF09uj). 
Finally, we note that if the CSM breakout explanation of very luminous SNe (Quimby et al. 2007) is correct, our analysis implies that these events should be accompanied by strong high energy X-ray emission. Lack off (Miller et al. 2009), or very weak (Smith et al. 2007), X-ray emission from some of these events challenges this interpretation.

B.K. is supported by NASA through Einstein Postdoctoral Fellowship awarded by the Chandra X-ray Center, which is operated by the Smithsonian Astrophysical Observatory for NASA under contract NAS8-03060. The research of E.W and N.S is partially supported by ISF, Minerva and PBC grants.

\section{References}

Balberg, S. \& Loeb, A. 2011, MNRAS, 478

Burrows, D. N., et al. 2005, Space Sc. Revs, 121, 165

Campana, S., et al. 2006, Nature, 442, 1008

Chevalier, R. A. \& Irwin, C. M. 2011, ApJ, 729, L6

Chevalier, R. A. \& Irwin, C. M. 2012, ApJ (Letters), 747, L17

Colgate, S. A. 1974, ApJ, 187, 321

Ensman, L. \& Burrows, A. 1992, ApJ, 393, 742

Epstein, R. I. 1981, ApJ, 244, L89

Falk, S. W. 1978, ApJ, 225, L133

Fan, Y.-Z., Zhang, B.-B., Xu, D., Liang, E.-W., \& Zhang, B. 2011, ApJ, 726, 32

Galama, T. J., Vreeswijk, P. M., van Paradijs, J., et al. 1998, Nature, 395, 670

Gehrels, N., et al. 2004, ApJ, 611, 1005

Hjorth, J., Sollerman, J., Møller, P., et al. 2003, Nature, 423, 847

Katz, B., Budnik, R., \& Waxman, E. 2010, ApJ, 716, 781

Katz, B., Sapir, N., \& Waxman, E. 2012, ApJ, 747, 147

Klein, R. I. \& Chevalier, R. A. 1978, ApJ, 223, L109

Kulkarni, S. R., Frail, D. A., Wieringa, M. H., et al. 1998, Nature, 395, 663

Lasher, G. J. \& Chan, K. L. 1979, ApJ, 230, 742

Malesani, D., Tagliaferri, G., Chincarini, G., et al. 2004, ApJ (Letters), 609, L5

Miller, A. A., et al. 2009, ApJ, 690, 1303

Moriya, T., Tominaga, N., Blinnikov, S. I., Baklanov, P. V., \& Sorokina, E. I. 2011, MNRAS, 415, 199

Murase, K., Thompson, T. A., Lacki, B. C., \& Beacom, J. F. 2011, Phys. Rev. D, 84, 043003

Nakar, E. \& Sari, R. 2010, ApJ, 725, 904

Ofek, E. O., et al. 2010, ApJ, 724, 1396

Quimby, R. M., Aldering, G., Wheeler, J. C., Höflich, P., Akerlof, C. W., \& Rykoff, E. S. 2007, ApJ (Letters), 668, L99

Rabinak, I. \& Waxman, E. 2011, ApJ, 728, 63

Sapir, N., Katz, B., \& Waxman, E. 2011, ApJ, 742, 36

Smith, N., et al. 2007, ApJ, 666, 1116

Smith, N. \& McCray, R. 2007, ApJ (Letters), 671, L17

Soderberg, A. M., Nakar, E., Berger, E., \& Kulkarni, S. R. 2006, ApJ, 638, 930

Soderberg, A. M., et al. 2008, Nature, 453, 469

Soderberg, A. M., Chakraborti, S., Pignata, G., et al. 2010, Nature, 463, 513

Svirski, G., Nakar, E., \& Sari, R. 2012, arXiv:1202.3437

Tan, J. C., Matzner, C. D., \& McKee, C. F. 2001, 20th Texas Symposium on relativistic astrophysics, 586, 638

Vergani, S. D., D'Avanzo, P., Levan, A. J., et al. 2010, GRB Coordinates Network, 10512, 1

Wang, X.-Y., Li, Z., Waxman, E., \& Mészáros, P. 2007, ApJ, 664, 1026

Waxman, E. \& Loeb, A. 2001, Phys. Rev. Lett., 87, 071101

Waxman, E., Mészáros, P., \& Campana, S. 2007, ApJ, 667, 351 